\title{
ÜBER DIE RESTKLASSENKÖRPER BEWERTETER PERFEKTER KÖRPER
}

\author{
MIKAO MORIYA
}

Die Struktur diskret bewerteter perfekter Körper ist bisher von H. Hasse, F. K. Schmidt, O. Teichmüller und E. Witt eingehend untersucht worden." Es ist schon von diesen Autoren bewiesen worden, dass der Restklassenkörper $\Re$ eines diskret perfekten Körpers $K$ stets in $K$ ein mit $\AA$ multiplikativ isomorphes Repräsentantensystem besitzt, und sogar, dass es im charakteristikgleichen Fall ein Repräsentantensystem $R$ von $\Re$ gibt, welches einen mit $\Re$ isomorphen Körper bildet. Dabei lässt sich $K$ als Potenzreihenkörper eines Primelementes aus $K$ mit Koeffizienten aus $R$ darstellen.

In der vorliegenden Arbeit betrachten wir den Fall, wo die Bewertung eines perfekten Körpers $K$ nicht notwendig diskret ist. Der Restklassenkörper $\cong$ von $K$ besitzt dann im allgemeinen kein multiplikativ abgeschlossenes Repräsentantensystem aus $K$; vielmehr muss man dabei maximale Teilkörper oder Gruppen aus $K$ bestimmen, welche bzw. Repräsentantensysteme der Teilkörper oder Gruppen mit gewissen Eigenschaften aus $\Re$ bilden.

§. Im folgenden bezeichnet $K$ durchweg einen Körper, welcher in bezug auf eine nicht-triviale Exponentenbewertung $w$ perfekt ist. ${ }^{2)} \quad$ Bekanntlich bildet die Gesamtheit $J$ aller derjenigen Elemente aus $K$, die in bezug auf $w$ nicht negative Werte besitzen, einen Ring (Bewertungsring in bezug auf $w$ ). Ferner bildet die Gesamtheit $p$ aller Elemente aus $K$, welche in bezug auf $w$ positive Werte besitzen, ein Primideal aus $J$. Der Restklassenring $\mathscr{R}=J / p$ ist ein Körper, welcher der Restklassenkörper von $K$ genannt ist. Eine Restklasse von $J$ nach $\mathfrak{p}$ nennt man auch eine Restklasse aus $\Re$. Ein beliebiges Element aus $K$, welches zu einer Restklasse aus $\Re$ gehört, heisst ein Vertreter dieser Restklasse:

Received September 4, 1951.

1) H. Hasse und F. K. Schmidt, Die Struktur diskret bewerteter Körper, Journ. f. reine u. angew. Math., 170 (1934).

O. Teichmüller, Über die Struktur diskret bewerteter perfekter Körper, Gött. Nachrichten, N.F. 1 (1936).

O. Teichmüller, Diskret bewertete perfekte Körper mit unvollkommenem Restklassenkőrper, Journ. f. reine u. angew. Math., 176 (1937).

E. Witt, Zyklische Körper und Algebren der Charakteristik $p$ vom Grade $p^{n}$, Journ. $f$. reine u. angew. Math., 176 (1937).

2) Für die allgemeine Theorie der Bewertung siehe das Buch von V. d. Waerden; Moderne Algebra, I. (1937), X. Kapt. Bewerteter Körper. 
Es liege nun eine nicht-leere Teilmenge $\mathfrak{M}$ von $\Re$ vor. Greift man dann aus jeder Restklasse aus $\mathfrak{M}$ einen einzigen Vertreter heraus, so heisse die Gesamtheit $M$ dieser Vertreter ein Repräsentantensystem von $\mathfrak{R}$ aus $K$. Wenn man dabei einem Element $\mathfrak{x}$ aus $\mathfrak{M}$ den Vertreter von $\mathfrak{x}$ aus $M$ zuordnet, so entsteht eine umkehrbar eindeutige Zuordnung $\varphi$ von $\mathfrak{M}$ auf $M$. Es sei insbesondere $\mathfrak{M}$ eine multiplikative Gruppe aus $\Re$ oder ein Teilkörper von $\Re$. Ist dann $M$ das isomorphe Bild von $\mathfrak{M}$ in bezug auf $\varphi$, so heisse $M$ mit $\mathfrak{M}$ gruppenoder körperisomorph.

Bekanntlich ist die Charakteristik $\chi(\Re)$ von $\Re$ stets gleich der Charakteristik $\%(K)$ von $K$, falls $\chi(K)$ eine Primzahl ist. Wenn aber $\chi(K)=0$ ist, so ist entweder $\chi(\Omega)=0$ oder $\chi(\Omega)=$ einer Primzahl.

§2. Wir betrachten zunächst den charakteristikgleichen Fall $\chi(K)=\chi(\AA)$. Dabei kann der Restklassenkörper $\Re$ auch unvollkommen sein. Wie man sich leicht überzeugt, ist dann der Primkörper von $K$ ein körperisomorphes Repräsentantensystem des Primkörpers von $\Re$. Nun sei $K_{\alpha} \subset K_{3} \subset \ldots \subset K_{\lambda} \subset \ldots$ eine Folge der Teilkörper von $K$ von der Art, dass jedes $K_{\lambda}$ ein körperisomorphes Repräsentantensystem eines Teilkörpers von $\Re$ ist. Bildet man jetzt die Vereinigung $V$ der obigen Körperfolge, so ist $V$ offenbar ein Teilkörper von $K$, und ferner existiert ein Teilkörper $\mathfrak{B}$ von $\mathfrak{R}$ derart, dass $V$ ein körperisomorphes Repräsentantensystem von $\mathfrak{B}$ ist. Nach dem bekannten Zorn's Lemma existiert also ein maximaler Teilkörper $K_{0}$ von $K$, welcher ein körperisomorphes Repräsentantensystem eines Teilkörpers $\Re_{0}$ von $\Re$ bildet. Es sei $K_{0}^{\prime}$ ein Teilkörper von $K$, welcher $K_{0}$ als einen echten Teilkörper enthält. Nehmen wir dann an, dass $z \mathfrak{u}$ jedem Element $x^{\prime}$ aus $K_{0}^{\prime}$ die $x^{\prime}$ enthaltende Restklasse aus $\mathscr{R}$ existiert, so existieren verschiedene Elemente $x^{\prime}, y^{\prime}$ aus $K_{0}^{\prime}$, welche $z$ u einer und derselben Restklasse aus $\mathscr{R}$ gehören. Denn sonst gäbe es einen Teilkörper $\mathscr{R}_{0}^{\prime}\left(\supset \Omega_{0}\right)$ von $\mathscr{I}$, der $K_{0}^{\prime}$ als ein körperisomorphes Repräsentantensystem besitzt ; dies steht aber im Widerspruch mit der Maximaleigenschaft über $K_{0}$. Daher ist $w\left(x^{\prime}-y^{\prime}\right)$ $>0$. Weil $x^{\prime}-y^{\prime} \neq 0$ ist, so existiert keine $\left(x^{\prime}-y^{\prime}\right)^{-1}$ enthaltende Restklasse aus $\Re$; d.h. jede echte Erweiterung von $K_{0}$ aus $K$ wird niemals ein Repräsentantensystem eines Teilkörpers von $\Re$.

Es sei $\mathfrak{b}$ ein über $\Re_{n}$ separables Element aus $\Re$. Dann genügt $\mathfrak{b}$ einer irreduziblen Gleichung $\sum_{i=0}^{m} a_{i} x^{i}=0$ in $\Re_{0}$. Bezeichnet man mit den $a_{i}(i=0,1, \ldots$, $m$ ) bzw. die in den $a_{i}$ enthaltenen Elemente aus $K_{n}$, so ist das Polynom $f(x)$ $=\sum_{i=0}^{m} a_{i} x^{i}$ in $K_{0}[x]$ separabel. Ist nämlich $f(x)$ reduzibel in $K_{0}[x]$, so existieren nicht triviale Polynome $g_{i}(x)(i=1,2)$ aus $K_{0}[x]$ mit $f(x)=g_{1}(x) g_{2}(x)$. Lässt man nun die sämtlichen Koeffizienten von $g_{1}(x), g_{2}(x)$ in die Restklassen von $\Omega_{0}$ übergehen, so entstehen aus den $g_{1}(x), g_{2}(x)$ bzw. die Polynome $\overline{g_{1}(x)}, \overline{g_{2}(x)}$ aus $\mathscr{R}_{0}[x]$, und es gilt in $\mathfrak{K}_{0}[x]$ : 


$$
\sum_{i=0}^{n} a_{i} x^{i}=\overline{g_{1}(x)} \overline{g_{2}(x)}
$$

d.h. $\sum_{i=0}^{m} a_{i} x^{i}$ ist entgegen der Annahme in $\mathscr{R}_{0}[x]$ reduzibel. Es muss also $f(x)$ in $K_{0}[x]$ irreduzibel sein. Die die Diskriminante von $f(x)$ enthaitende Restklasse aus $\Re$ ist gleich der Diskriminante des separablen Polynomes $\sum_{i=0}^{m} a_{i} x^{i}$, also ist von der Nullklasse verschieden. Somit ist gezeigt, dass $f(x)$ in $K_{0}[x]$ separabel ist.

Nun gilt für ein Element $b$ aus $\mathfrak{b}$ die Kongruenz:

$$
f(b) \equiv 0 \quad \bmod p ;
$$

d.h. es existiert ein Polynom $h(x)$ mit Koeffizienten aus $J$ derart, dass

$$
f(x) \equiv(x-b) h(x) \quad \bmod \mathfrak{p}
$$

ist. Nach dem bekannten Hensel's Lemma beweist man die Existenz einer Nullstelle $\beta$ von $f(x)$ aus $J$, für die

$$
\beta \equiv b \quad \bmod p
$$

gilt. Adjungiert man $\beta z \mathrm{u} K_{0}$, so ist der Körper $K_{0}(\beta)$ durch einen solchen Isomorphismus $\Phi$ auf $\Omega_{0}(b)$ abgebildet, dass bei Anwendung von $\Phi$ der Isomorphismus von $K_{0}$ auf $\Omega_{0}$ beibehalten und $\Phi(\beta)=\mathfrak{b}$ ist. Wegen der Maximaleigenschaft über $K_{0}$ muss $K_{0}(\beta) \subseteq K_{0}$ sein; d.h. die $\beta$ enthaltende Restklasse $\mathfrak{b}$ aus $\mathscr{A}$ ist bereits ein Element aus $\Omega_{0}$. Somit ist bewiesen, dass jedes über $\Omega_{0}$ separable Element aus $\Re$ stets in $\Re_{0}$ enthalten ist; d.h. $\Re_{0}$ ist in $\Re_{\text {separabel-abgeschlossen. }}$

Es sei jetzt $t$ ein uber $\Omega_{0}$ transzendentes Element aus $\mathscr{R}$. Ist dann $t$ ein Element aus 1 , so ist $t$ auch über $K_{0}$ transzendent. Denn gälte für die nicht sämtlich verschwindenden Elemente $a_{0}, a_{1}, \ldots, a_{n}$ aus $K_{0}$

$$
a_{0}+a_{1} t+\ldots+a_{n} t^{n}=0
$$

so würde daraus folgen :

$$
\bar{a}_{0}+\bar{a}_{1} t+\ldots+\bar{a}_{n} t^{n}=\overline{0},
$$

wo die $\bar{a}_{0}, \bar{a}_{1}, \ldots, \bar{a}_{n}$ bzw. die $a_{0}, a_{1}, \ldots, a_{n}$ enthaltenden Restklassen aus $\Re_{0}$ bezeichnen. Dies ist aber ein Widerspruch. Der Körper $K_{0}(t)$ ist offenbar ein körperisomorphes Repräsentantensystem von $\mathcal{K}_{0}(t)$. Dies steht aber wieder mit der Maximaleigenschaft über $K_{0}$ im Widerspruch. Ist also $\mathfrak{c}$ ein nicht $z^{\prime} 1$ $\mathfrak{R}_{0}$ gehöriges Element aus $\mathscr{R}$, so muss $c$ stets über $\mathscr{R}_{0}$ inseparabel sein. Daraus erhält man

SATZ 1. Ist $\%(K)=\%(\Omega)=0$, so existiert in $K$ stets ein körperisomorphes Repräsentantensystem von $\Omega$.

Wir wenden uns jetzt zum Fall $\%(K)=\chi(\mathfrak{R})=p \neq 0$. Ist dann c ein nicht 
zu $\Re_{0}$ gehöriges Element aus $\Re$, so existiert eine Potenz $\mathfrak{c}^{p^{f}}$ von $\mathfrak{c}$ mit $f \geqq 1$ derart, dass $c^{p^{f}}$ über $\Re_{0}$ separabel ist. Weil nach dem oben Bewiesenen $c^{p f}$ zu $\Re_{0}$ gehört, so ist $\mathfrak{c}$ über $\Omega_{0}$ rein-inseparabel. Somit ist bewiesen :

SATZ 2. Ist $\chi(K)=\chi(\Re)=p \neq 0$, so existiert in $K$ ein maximaler Teilkörper $K_{0}$, welcher ein körperisomorphes Repräsentantensystem eines Teilkörpers $\mathfrak{H}_{0}$ von $\Re$ ist. Jede echte Erweiterung von $K_{0}$ aus $K$ wird niemals ein Repräsen-

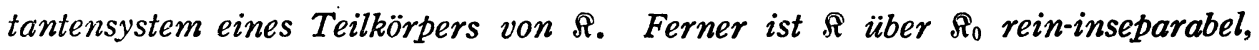
wenn $\Re \neq \Re_{0}$ ist.

Bemerkung. Obwohl $K_{0}$ in $K$ maximal ist, so ist $\Re_{0}$ doch nicht in $\Re$ maximal; d.h. es kann in $\Re$ eine echte Erweiterung von $\Re_{0}$ geben, welche ein körperisomorphes Repräsentantensystem aus $K$ besitzt.

Setzt man aber voraus, dass jeder Teilkörper von $\Re$ höchstens ein körperisomorphes Repräsentantensystem aus $K$ besitzt, so ist $\mathfrak{\Omega}_{0}$ in $\Re$ maximal; d.h. jede echte Erweiterung von $\Re_{0}$ aus $\Re$ besitzt kein körperisomorphes Repräsentantensystem aus $K$. Besässe nämlich eine echte Erweiterung $\Omega_{1}$ von $\Omega_{0}$ aus $\mathscr{R}$ ein körperisomorphes Repräsentantensystem $K_{1}$ aus $K$, so wäre nach Voraussetzung $K_{1} \neq K_{0}$, was aber ein Widerspruch ist.

ZuSATZ zu SATZ 2. Wenn jeder Teilkörper von $\Re$ höchstens ein körperisomorphes Repräsentantensystem aus $K$ besitzt, so existiert ein maximaler Teilkörper $\Re_{0}$ von $\Re$ derart, dass $\Re_{0}$ ein körperisomorphes Repräsentantensystem aus $K$ besitzt, aber jede echte Erweiterung von $\Omega_{0}$ aus $\Re$ kein körperisomorphes $R e-$ präsentantensystem aus $K$ besitzt.

Wir betrachten nun den charakteristikungleichen Fall $\chi(K) \neq \chi(\Omega)$, und bezeichnen mit $p$ die Charakteristik von $\Omega$. Ferner bezeichnen wir mit $K^{*}$ bzw. $\Omega^{*}$ die multiplikative Gruppe aller von Null verschiedenen Elemente aus $K$ bzw. $\Omega$.

Ist nun $i(p-1 \geqslant i \geqslant 0)$ eine ganze rationale Zahl und $\bar{i}$ die $i$ enthaltende Restklasse aus $\mathfrak{R}$, so genügt $\bar{i}$ der Gleichung

$$
x^{p-1}-\overline{1}=\overline{0} \text {. }
$$

Mit Hilfe des Hensel's Lemmas kann man leicht beweisen, dass die Gleichung $x^{p-1}-1=0 p-1$ verschiedene Wurzeln aus $K$ besitzt, welche im ganzen mod $p$ mit den Zahlen $1,2, \ldots, p-1$ kongruent sind; d.h. $K^{*}$ enthält die zyklische Gruppe $Z_{p-1}$ aller $(p-1)$-ten Einheitswurzeln, welche ein gruppenisomorphes Repräsentantensystem der Untergruppe $\{\overline{1}, \overline{2}, \ldots, \overline{p-1}\}$ aus $\Omega^{*}$ ist. Daher existiert nach dem Zorn's Lemma eine maximale Untergruppe $G_{0}$ von $K^{*}$, welche ein gruppenisomorphes Repräsentantensystem einer Untergruppe $\mathbb{S}_{0}$ von $\Omega^{*}$ bildet.

Nun sei $\mathfrak{b}$ ein Element aus $\Re^{*}$, dessen Ordnung $n$ nach $\mathscr{S}_{0}$ zu $p$ prim ist. 
Dann existiert ein Element $\mathfrak{a}$ aus $\mathfrak{S}_{0}$ mit $\mathfrak{b}^{n}=\mathfrak{a}$. Da es in $G_{0}$ ein Element $a \in \mathfrak{a}$ gibt, so betrachten wir die Gleichung $x^{n}-a=0$ in $K$. Offenbar genügt ein Element $b$ aus $\mathfrak{b}$ der Kongruenz:

$$
x^{n}-a \equiv 0 \quad \bmod \mathfrak{p} .
$$

Wie man leicht bestätigt, besitzt wegen $p+n$ die obige Kongruenz keine mehrfachen Wurzeln. Hieraus schliesst man leicht nach dem Hensel's Lemma, dass es in $K$ eine Wurzel $\beta$ von $x^{n}-a=0$ mit $\beta \equiv b \bmod p$ gibt; es gilt also $\beta^{n}=a$. Weil die Ordnung $\mathfrak{b}$ nach $\left(\mathscr{S}_{0}\right.$ gleich $n$ ist, so ist die Ordnung von $\beta$ nach $G_{0}$ auch $n$. Betrachtet man jetzt die Menge $G_{1}=\left\{G_{0}, G_{0} \beta, \ldots, G_{0} \beta^{n-1}\right\}$, so ist $G_{1}$ ein gruppenisomorphes Repräsentantensystem einer Untergruppe von $K^{*}$. Es muss also $G_{0}=G_{1}$ sein ; d.h. es ist $n=1$.

Nun sei $t$ ein Element aus $\AA^{*}$, dessen Ordnung nach $\mathbb{S}_{0}$ gleich 0 ist; d.h. eine Poten $\mathrm{t}^{m}$ von $\mathrm{t}$ gehöre dann und nur dann $z \mathrm{u} \$_{0}$, wenn $m=0$ ist. Ist dann $t$ ein zu $t$ gehöriges Element aus $K$, so bestätigt man leicht, dass die Ordnung von $t$ nach $G_{0}$ auch 0 ist. Ferner beweist man ohne Schwierigkeit, dass die Menge $G_{2}=\left\{G_{0} t^{ \pm i} ; i=0,1, \ldots\right\}$ ein gruppenisomorphes Repräsentantensystem einer Untergruppe von $\Re^{*}$ bildet, was aber mit der Maximaleigenschaft über $G_{0}$ im Widerspruch steht. Aus dem oben Gezeigten schliesst man sofort, dass ein nicht zu $\mathbb{S}_{0}$ gehöriges Element aus $\mathfrak{K}^{*}$ von einer Ordnung $p^{f}(f \gtrsim 1)$ ist.

SATZ 3. Es sei $\chi(K) \neq \chi(\Omega)=\not(\neq 0)$. Dann existiert in $K^{*}$ eine maximale Untergruppe $G_{0}$ von der Art, dass $G_{0}$ ein gruppenisomorphes Repräsentantensystem einer Untergruppe $\mathbb{S}_{0}$ von $\Re^{*}$ bildet und jede echte Obergruppe von $G_{0}$ aus $K^{*}$ niemals ein gruppenisomorphes Repräsentantensystem einer Untergruppe von $\Re^{*}$ wird. Ferner ist die Ordnung eines nicht zu $\leftrightarrow_{0}$ gehörigen Elementes aus $\AA^{*}$ nach $\mathbb{S}_{0}$ eine Potenz von $p$.

Bemerkung. Wie bei Satz 2 kann man ohne Schwierigkeit folgenden Satz beweisen :

ZuSATZ Zu SATZ 3. Besitzt jede Uniergruppe aus $\Omega^{*}$ höchstens ein gruppenisomorphes Repräsentantensystem aus $K^{*}$, so ist $\$_{0}$ eine maximale Untergruppe aus $\mathfrak{\Omega}^{*}$, welche ein gruppenisomorphes Repräsentaniensystem aus $K^{*}$ besitzt.

§3. In diesem Paragraphen beschränken wir uns auf den Fall, wo der Restklassenkörper $\mathfrak{R}$ vollkommen von einer Primzahlcharakteristik $p$ ist. Bekanntlich besitzt jede Restklasse aus $\Re$ stets ihre $p^{i}$-ten Wurzeln $(i=1,2, \ldots)$ in $\Re$.

Zunächst betrachten wir den charakteristikgleichen Fall. Da der Primkörper von $K$ vollkommen und ein körperisomorphes Repräsentantensystem des Primkörpers von $\Omega$ ist, so beweist man leicht mit Hilfe des Zorn's Lemmas die 
Existenz eines maximalen vollkommenen Teilkörpers $K_{0}$ aus $K$ von der Art, dass $K_{0}$ ein körperisomorphes Repräsentantensystem eines Teilkörpers $\Re_{0}$ aus $\mathfrak{A}$ bildet.

Ist nun $\mathfrak{b}$ ein über $\Re_{0}$ algebraisches Element aus $\mathscr{R}$, so muss $\mathfrak{b}$ über $\Re_{0}$ separabel sein, weil $\Re_{0}$ vollkommen ist. Ebenso wie in $\S 2$ kann man leicht beweisen, dass es ein Element $\beta$ aus $\mathfrak{b}$ gibt, so dass $K_{0}(\beta)$ ein körperisomorphes Repräsentantensystem von $\Re_{0}(\mathfrak{b})$ ist. $\mathrm{Da} K_{0}(\beta)$ über $K_{0}$ algebraisch ist, so ist es vollkommen; wegen der Maximaleigenschaft über $K_{0}$ muss $K_{0}(\beta) \cong K_{0}$ sein, und infogedessen ist $\mathfrak{b} \in \Re_{0}$. Somit ist bewiesen :

SATz 4. Es sei $\%(K)=\%(\Re)=p \neq 0$ und $\Re$ vollkommen. Dann enthält $K$ einen maximalen vollkommenen Körper $K_{0}$ von der Art, dass $K_{0}$ ein körperisomorphes Repräsentantensystem eines Teilkörpers $\mathfrak{R}_{0}$ von $\Re$ bildet. Dabei ist $\Re_{0}$ in $\Re$ algebraisch abgeschlossen.

Wenn insbesondere $\Re$ absolut-algebraisch ist, so ist bekanntlich $\Re$ vollkommen, und jedes Element aus $\Re$ ist stets über dem Primkörper von $\Re$ algebraisch. Hieraus folgi

ZuSATZ ZU SATZ 4. Es sei $\chi(K)=\chi(\Omega)=p \neq 0$ und $\Re$ absolut-algebraisch. Dann enthält $K$ stets ein körperisomorphes Repräsentantensystem von $\Re$.

Nun wollen wir folgenden Satz beweisen :

SATZ 5. Ist $\%(K)=\%(\Re)=p \neq 0$ und $\Re$ vollkommen, so existiert in $K$ dann und nur dann ein körperisomorphes Repräsentantensystem von $\Re$, wenn für jede Restklasse c aus $\Re$ der Durchschnitt $\bigcap_{i=0}^{\infty} \mathfrak{R}_{i}(\mathrm{c})$ nicht leer ist. Dabei bezeichnet $\mathfrak{M}_{i}(\mathfrak{c})$ die Menge der $p^{i}$-ten Potenzen aller Elemente aus $\mathfrak{c}^{p^{-i}}(i \geqq 0)$.

Beweis. Wenn ein Teilkörper $K_{0}$ von $K$ ein körperisomorphes Repräsentantensystem von $\mathscr{R}$ ist, so gibt es zu einer Restklasse $c$ aus $\mathbb{R}$ ihren (einzigen) Vertreter $c$ aus $K_{0}$. Da $K_{0}$ vollkommen ist, so besitzt $c$ in $K_{0}$ seine $p^{i}$-te Wurzel $c^{p^{-i}}(i \geqslant 0)$, und $c^{p^{-i}}$ ist der Vertreter von $c^{p^{-i}}$ aus $K_{0}$. Also gehört $c z u$ $\mathfrak{M}_{i}(\mathfrak{c})$. Hieraus folgt ohne weiteres, dass $\bigcap_{i=0}^{\infty} \mathfrak{M}_{i}(\mathfrak{c})$ nicht leer ist.

Umgekehrt sei für jede Restklasse c aus $\mathbb{R} \bigcap_{i=0}^{\infty} \mathfrak{M}_{i}(\mathrm{c})$ nicht leer. Dann betrachten wir einen maximalen vollkommenen Teilkörper $K_{0}$ von $K$, welcher mit einem in $\mathfrak{K}$ algebraisch-abgeschlossenen Teilkörper $\Re_{0}$ körperisomorph ist (Satz 4). Ist nun $t$ ein über $\Re_{0}$ transzendentes Element aus $\Re$, so existiert nach Voraussetzung ein Element $t$ aus $\bigcap_{i=0}^{\infty} \mathfrak{M}_{i}(\mathrm{t})$. Wie leicht bestätigt, ist $t$ über $K_{0}$ transzendent. Ferner existiert in jedem $\mathrm{t}^{p^{-i}}$ ein Element $t_{i}$ aus $K$ mit $t_{i}^{p^{i}}=t$. Da $\chi(K)=p$ ist, so gilt für jedes $i \geqslant 0$ :

$$
t_{i}=t_{i+1}^{p}
$$


wo $t_{0}=t$ gesetzt ist. Bildet man nun den Vereinigungskörper $K_{1}$ von den Körpern $K_{0}\left(t_{i}\right)(i=0,1, \ldots)$, so ist $K_{1}$ ersichtlich ein vollkommener Teilkörper von $K$, weil $K_{0}$ vollkommen ist. Da für jeden Index $i(\gg 0) K_{0}\left(t_{i}\right)$ ein körperisomorphes Repräsentantensystem von $\mathscr{R}_{0}\left(\mathrm{t}^{p^{-i}}\right)$ aus $\mathscr{R}$ ist, so bildet $K_{1}$ ein körperisomorphes Repräsentantensystem des Vereinigungskörpers von den $\Omega_{0}\left(1^{t^{-i}}\right)$ $(i=0,1, \ldots)$. Dies steht aber mit der Maximaleigenschaft über $K_{0}$ im Widerspruch. Es muss also $\Re_{0}=\Re$ sein, w.z.b.w.

Jetzt wenden wir uns zum charakteristikungleichen Fall, und wir bedienen uns aller Bezeichnungen im vorigen Paragraphen. Die zyklische Gruppe $Z_{p-1}$ aller $(p-1)$-ten Einheitswurzeln aus $K^{*}$ ein gruppenisomorphes Repräsentantensystem einer Untergruppe von $\mathfrak{R}^{*}$. Ferner ist $Z_{p-1}=Z_{p-1}^{p}$, wo $Z_{p-1}^{p}$ die Menge aller $p$-ten Potenzen der Elemente aus $Z_{p-1}$ bezeichnet. Nach dem Zorn's Lemma existiert eine maximale Untergruppe $G_{0}$ mit $G_{0}=G_{0}^{p}$ aus $K^{*}$, welche ein gruppenisomorphes Repräsentantensystem einer Untergruppe $\mathbb{S}_{0}$ von $\mathfrak{K}^{*}$ bildet.

Es sei $\mathfrak{b}$ ein Element aus $\mathfrak{R}^{*}$, dessen eine Potenz $\mathfrak{b}^{m}(m>0)$ zu $\mathscr{G}_{0}$ gehört. Dann setzen wir $m=m_{0} p^{f}$, wo $\left(m_{0}, p\right)=1$ ist, und $\mathfrak{a}=\mathfrak{b}^{m_{0}}$. Weil aber $a^{p^{f}}$ ein Element aus $G_{0}$ ist, so gibt es in $G_{0}$ seinen Vertreter $a_{f}$. Wegen $G_{0}=G_{0}^{p}=\ldots$ $=G_{0}^{p f}$ existiert ein Element $a_{1}$ aus $G_{0}$ mî́ $a_{f}=a_{1}^{p f}$. Es gilt also für die $a_{1}$ enthaltende Restklasse $a_{1}$ aus $\Re$ :

$$
\mathfrak{a}^{p f}=\mathfrak{a}_{1}^{p f}
$$

weil $\chi(\Re)=p$ ist, so folgt aus der obigen Gleichung

$$
\mathfrak{a}=a_{1} \in \mathbb{S}_{0} \text {. }
$$

$\mathrm{Da} \mathfrak{b}^{m_{0}}=\mathfrak{a} z \mathrm{zu} \mathscr{G}_{0}$ gehört und $m_{0} \mathrm{zu} p$ prim ist, so ist die Ordnung von $\mathfrak{b}$ nach $\mathscr{G}_{0}$ prim zu $p$. Wie in $\S 2$ kann man leicht beweisen, dass $\mathfrak{b}$ bereits $z \mathfrak{u} \mho_{0}$ gehören muss. Somit ist bewiesen :

SATZ 6. Es sei $\chi(K) \neq \chi(\Re)=p$ und $\Re$ vollkommen. Dann existiert eine maximale Untergruppe $G_{0}$ von $K^{*}$ mit $G_{0}=G_{0}^{p}$ von der Art, dass $G_{0}$ ein gruppenisomorphes Repräsentantensystem einer Untergruppe $\mathfrak{G}_{0}$ von $\Re^{*}$ bildet. Dabei besitzt die Faktorgruppe $\AA^{*} / \bigotimes_{0}$ ausser dem Einheitselement kein Element von einer positiven Ordnung.

Ist insbesondere $\Re$ absolut-algebraisch, so ist jedes Element aus $\AA^{*}$ von einer positiven Ordnung. Daraus folgt nach Satz 6 :

ZuSATZ ZU SATZ 6. Es sei $\chi(K) \neq \chi(\Omega)=p$ und $\Omega$ absolui-algebraisch. Dann besitzt $K^{*}$ ein gruppenisomorphes Repräsentantensystem von $\AA^{*}$.

Wir beweisen nun folgenden

SATZ 7. Ist $\chi(K) \neq \chi(\Re)=p$ und $\Re$ vollkommen, so enthält $K^{*}$ dann und 
nur dann ein gruppenisomorphes Repräsentantensystem von $\AA^{*}$, wenn aus den $p^{i}$-ten Wurzeln $c^{p^{-i}}(i=0,1, \ldots)$ einer beliebigen Restklasse $c$ aus $\Omega^{*}$ stets die Vertreter $c_{i}$ derart herausgegriffen werden können, dass für jeden Index $i(\gtrsim 0)$ die Relation

$$
c_{i}=c_{i+1}^{p}
$$

gilt.

Beweis. Es sei $G_{0}$ ein gruppenisomorphes Repräsentantensystem von $\mathfrak{K}^{*}$ aus $K^{*}$. Dann ist $\Re^{*}=\Re^{* p}$, wei] $\Re$ vollkommen ist. Dabei bezeichnet $\Re^{* \phi}$ die Menge der $p$-ten Potenzen aller Elemente aus $\mathfrak{R}^{*}$. Weil $G_{0}$ mit $\Re^{*}$ gruppenisomorph ist, so gilt $G_{0}=G_{0}^{\phi}$. Ist nun c ein Element aus $\AA^{*}$, so gibt es in $G_{0}$ den Vertreter $c_{0}$ von c. Wegen $G_{0}=G_{0}^{p}$ existiert $z \mathrm{u}$ jedem Index $i(\geqslant 0)$ ein Element $c_{i}$ aus $G_{0}$ mit $c_{i}=c_{i+1}^{p}$. Offenbar ist $c_{1}$ der Vertreter von $c^{p^{-1}}$ aus $G_{0}$. Durch vollständige Induktion beweist man, dass $c_{i}$ der Vertreter von $c^{p^{-i}}$ aus $G_{0}$ ist.

Umgekehrt sei die Voraussetzung des Satzes erfüllt. Dann betrachten wir eine maximale Untergruppe $G_{0}$ mit $G_{0}=G_{0}^{p}$ von $K^{*}$ von der Art, dass $G_{0}$ ein gruppenisomorphes Repräsentantensystem einer Untergruppe $\mathscr{G}_{0}$ von $\Re^{*}$ bildet. Dabei hat in der Faktorgruppe $\mathfrak{\Re}^{*} / \mathscr{S}_{0}$ nur das Einheitselement eine positive Ordnung (Satz 6).

Es sei $t$ ein Element aus $\Omega^{*}$, welches in bezug auf $\mathscr{S}_{0}$ die Ordnung 0 hat. Dann kann man nach Voraussetzung aus den Restklassen $t^{p^{-i}}(i=0,1, \ldots)$ bzw. diejenigen Elemente $t_{i}$ herausgreifen, für die Relationen $t_{i}=t_{i+1}^{p}(i=0,1, \ldots)$ gelten. Offenbar ist jedes $t_{i}(i=0,1, \ldots)$ in bezug auf $G_{0}$ von der Ordnung 0 . Bildet man nun die Gruppe $H_{i}=\left\{G_{0} t_{i}^{ \pm \nu} ; \nu=0,1, \ldots\right\}$, so ist $H_{i}$ in $\AA^{*}$ gruppenisomorph abgebildet. Ferner ist

$$
H_{i}=H_{i+1}^{p} \subset H_{i+1},
$$

weil $G_{0}=G_{0}^{p}$ und $t_{i}=t_{i+1}^{p}$ sind. Offenbar ist die Vereinigungsmenge $G_{1}$ von den $H_{i}(i=0,1, \ldots)$ eine Untergruppe von $K^{*}$ mit $G_{1}=G_{1}^{\phi}$ und in $\Re^{*}$ gruppenisomorph abgebildet. Dies ist aber ein Widerspruch, weil $G_{1}$ eine echte Obergruppe von $G_{0}$ ist. Daher muss $\left(\$_{0}=\Re^{*}\right.$ sein, w.z.b.w.

Wir nehemn nun an, dass $K$ keine primitive $p$-te Einheitswurzel enthält und für jede Restklasse $c$ aus $\Omega^{*}$ stets $\bigcap_{i=0}^{\infty} \mathfrak{M}_{i}(c)$ nicht leer ist. Ist nun $c$ ein Element aus $\bigcap_{i=0}^{\infty} \mathfrak{M}_{i}(\mathfrak{c})$, so existiert zu jedem $i(\gg 0)$ ein Element $c_{i}$ aus $\mathfrak{c}^{p^{-i}}$ derart, dass

$$
c=c_{0}=c_{i}^{p^{i}}
$$

ist. Da offenbar $c_{i}^{p^{i}}=\left(c_{i+1}^{p}\right)^{p^{i}}$ ist und $K$ keine primitive $p$-te Einheitswurzel enthält, so schliesst man ohne Schwierigkeit, dass $c_{i}=c_{i+1}^{p}(i \geqslant 0)$ ist. Hieraus 
folgt :

Zusatz 1 zu Satz 7. Es sei $\chi(K) \neq \chi(\Re)=p$ und $\Re$ vollkommen. Enthält dann $K^{*}$ keine primitive $p$-te Einheitswurzel, so existiert in $K^{*}$ ein gruppenisomorphes Repräsentantensystem von $\AA^{*}$, wenn für jede Restklasse c aus $\Pi^{*}$ stets $\bigcap_{i=0}^{\infty} \mathfrak{M}_{i}(\mathrm{c})$ nicht leer ist.

Enthält aber für jede Restklasse c aus $\Omega^{*}$ der Durchschnitt $\bigcap_{i=0}^{\infty} \mathfrak{M}_{i}(\mathrm{c})$ genau ein Element, so betrachten wir für eine beliebige, nicht negative Zahl $\nu$ den Durchschnitt

$$
\left.\bigcap_{i=0}^{\infty} 9\right\}_{i}\left(c^{p^{-\nu}}\right) \text {. }
$$

Nach Voraussetzung enthält dieser Durchschnitt ein einziges Element $c_{\nu}$ aus $K$. Wegen $c_{\nu+1} \in \mathfrak{M}_{i}\left(c^{p^{-\nu-1}}\right)$ ist offenbar

$$
c_{\nu+1}^{p} \in \mathfrak{D} \lambda_{i+1}\left(\mathfrak{c}^{p^{-\nu}}\right) .
$$

Hieraus folgt sofort:

$$
c_{\nu+1}^{p}=\bigcap_{i=0}^{\infty} \mathfrak{M}_{i+1}\left(\mathfrak{c}^{p^{-\nu}}\right)=\bigcap_{i=0}^{\infty} \mathfrak{M}_{i}\left(\mathfrak{c}^{p^{-\nu}}\right)=c_{\nu} .
$$

Aus Satz 7 folgt also

ZuSATZ 2 zU SATZ 7. Es sei $\chi(K) \neq \chi(\Re)=p$ und $\Re$ vollkommen. Besitzt dann fïr jede Restklasse c aus $\Re^{*}$ der Durchschnitt $\bigcap_{i=0}^{\infty} i_{i}(\mathrm{c})$ genau ein Element, so existiert (genau) eine Untergruppe aus $K^{*}$, welche mit $\AA^{*}$ gruppenisomorph ist.

Bemerkung. Im Fall, wo die Bewertung $w$ von $K$ diskret ist, so greifen wir aus den $p^{-i}$-ten Wurzeln $c^{p^{-i}}$ einer beliebigen Restklasse $c$ aus $\mathfrak{R}$ bzw. die (beliebigen) Vertreter $a_{i}$ heraus. Da $c^{p^{-i}} \supseteqq \mathfrak{M}_{1}\left(c^{p^{-(i+1)}}\right)$ ist, so gilt:

$$
a_{i} \equiv a_{i+1}^{p} \quad \bmod \pi \quad(i \geqslant 0),
$$

wo $\pi$ ein Primelement von $w$ bezeichnet. Dann gilt offenbar:

$$
a_{i}^{p^{i}} \equiv a_{i+1}^{p^{i+1}} \quad \bmod \pi^{i+1} .
$$

Also konvergiert die Folge $a_{n}, a_{1}^{p}, \ldots, a_{i}^{p^{i}}, \ldots z$ u einem Element $c_{0}$ aus $J$. Da offenbar $a_{i}^{p^{i}} \in \mathfrak{M}_{i}(\mathrm{c}) \subseteq \mathrm{c}$ ist, so ist $c=c_{0}$ ein Element aus c. Ebenso beweist man, dass die Folge $a_{1}, a_{2}^{p}, \ldots, a_{i+1}^{p i}, \ldots$ zu einem Element $c_{1}$ aus $c^{p^{-1}}$ konvergiert. Da aber $c_{1}^{p}$ das Grenzelement der konvergenten Folge $a_{1}^{p}, a_{2}^{p^{2}}, \ldots$, $a_{i+1}^{p^{i+1}}, \ldots$ ist, so erhält man :

$$
c=c_{0}=c_{1}^{p} \text {. }
$$

Durch vollständige Induktion beweist man leicht, dass es in jedem $c^{p^{-i}}$ ein Ele- 
ment $c_{i}$ mit $c_{i}=c_{i+1}^{p}$ gibt. Ferner gilt:

d.h. der Durchschnitt $\bigcap_{i=0}^{\infty} \mathfrak{M}_{i}(c)$ ist nicht leer.

$$
c=c_{0}=c_{1}^{p}=\ldots=c_{i}^{p^{i}}=\ldots ;
$$

Nun gibt es für ein Element $c^{\prime}$ aus $\bigcap_{i=0}^{\infty} \mathbb{M}_{i}(\mathfrak{c})$ ein Element $c_{i}^{\prime}$ aus $c^{p^{-i}}$ mit $c^{\prime}=c_{i}^{\prime p^{2}}$. Da aber für jeden Index $i(\geq 0)$ stets die Kongruenz

$$
c_{i} \equiv c_{i}^{\prime} \quad \bmod \pi
$$

besteht, so erhält man für eine beliebige natürliche Zahl $m$ stets

$$
c=c_{m}^{p^{m}} \equiv c_{m}^{\prime p^{m}}=c^{\prime} \quad \bmod \pi^{m}
$$

d.h. es ist $c=c^{\prime}$. Der Durchschnitt $\bigcap_{i=0}^{\infty} \mathbb{M}_{i}(c)$ enthält also ein einziges Element. Daraus schliesst man im charakteristikgleichen Fall nach Satz 5 , dass es in $K$ genau ein körperisomorphes Repräsentantensystem von $\mathbb{R}$ gibt. Im charakteristikungleichen Fall existiert aber nach Satz 7 ein einziges gruppenisomorphes Repräsentantensystem $G$ von $\Re$. Die Gruppe $G$ bildet mit der Null zusammen ein multiplikativ abgeschlossenes System $R$, und $R$ ist mit $\Re$ multiplikativ isomorph. Zusammenfassend die beiden obigen Tatsachen, erhält man einen Teil der am Anfang erwähnten Tatsache:

Ist $K$ ein diskret bewerteter perfekter Körper und $\Re$ vollkommen von einer Primzahlcharakteristik $p$, so gibt es in $J$ ein einziges, mit $\mathbb{R}$ multiplikativ isomorphes Repräsentantensystem $R$ von $\mathfrak{R}$ mit $R^{p}=R$. Wenn $K$ die Charakteristik $p$ hat, so ist $R$ mit $\Re$ körperisomorph.

$\S 4$. In diesem Paragraphen wollen wir durch ein Beispiel zeigen, dass es im charakteristikgleichen Fall einen perfekten Körper $K$ mit dem vollkommenen Restklassenkörper $\Re$ gibt, welcher kein körperisomorphes Repräsentantensystem von $\Re$ besitzt.

$\mathrm{Zu}$ diesem $\mathrm{Zweck}$ betrachten wir einen vollkommenen Körper $k_{0}$ von einer Primzahlcharakteristik $p$, und eine einfache transzendente Erweiterung $k_{0}(t)$ von $k_{0}$ mit einem über $k_{0}$ transzendenten Element $t$. Ferner betrachten wir für eine zu $p$ prime natürliche Zahl $q(>1)$ eine additive Gruppe $W$ aller derjenigen rationalen Zahlen, die, dargestellt als reduzierter Bruch, lauter Potenzen von $\boldsymbol{q}$ als Nenner besitzen. Dann bezeichnen wir mit $I(t, x)$ den Integritätsbereich aller verallgemeinerten Polynome $a_{0}(t)+a_{1}(t) x^{\alpha_{1}}+\ldots+a_{n}(t) x^{\alpha_{n}}$ von $x$ mit Koeffizienten $a_{i}(t)(i=0,1, \ldots, n)$ aus $k_{0}(t)$, wo $0<\alpha_{1}<\ldots<\alpha_{n}\left(\alpha_{i} \in W\right)$ ist. Jedes Element $y \neq 0$ aus dem Quotientenkörper $Q$ von $I(t, x)$ ist von der Form:

$$
y=x^{\alpha} \frac{a_{0}(t)+a_{1}(t) x^{\alpha_{1}}+\ldots+a_{n}(t) x^{\alpha_{n}}}{b_{0}(t)+b_{1}(t) x^{\beta_{1}}+\ldots+b_{m}(t) x^{\beta_{m}}} .
$$

Dabei sind $a_{0}(t)+a_{1}(t) x^{\alpha_{1}}+\ldots+a_{n}(t) x^{\alpha_{n}}$ und $b_{0}(t)+b_{1}(t) x^{\beta_{1}}+\ldots+b_{m}(x) x^{\beta_{m}}$ 
Polynome aus $I(t, x)$ mit $a_{0}(t) b_{0}(t) \neq 0$. Ferner heisst die Zahl $\alpha$ der Exponent von $y$, weil $\alpha$ in der obigen Darstellung durch $y$ eindeutig bestimmt ist. Setzt man nun $w(y)=\alpha,{ }^{3)}$ so definiert $w$, wie leicht bestätigt, eine Exponentenbewertung von $Q$ mit $W$ als der Wertgruppe. Offenbar ist dabei ein von Null verschiedenes Element aus $k_{0}(t)$ stets die Bewertung 0 . Wir bilden nun die perfekte Hülle $K$ von $Q$ in bezug auf $w$ und bezeichnen mit $J$ den Bewertungsring von $K$ in bezug auf $w$. Bezeichnet nun $\mathfrak{p}$ das zu $w$ gehörige Primideal aus $J$, so ist der Restklassenkörper $\Omega=J / p$ von $K$ mit $k_{0}(t)$ körperisomorph.

Nun seien $\rho_{1}=x^{q^{-1}}, \rho_{2}=\rho_{1}+x^{p q^{-2}}, \ldots, \rho_{n}=\rho_{n-1}+x^{p^{n-1} q^{-n}}, \ldots$ gesetzt. Bezeichnet man dann mit $\overline{t+\rho_{n}}$ die $t+\rho_{n}$ enthaltende Restklasse aus $\Re$, so ist das Polynom $F(X)=X^{p^{n}}-\overline{t+\rho_{n}}$ irreduzibel in $K[X]$. Angenommen, es wäre $F(X)$ reduzibel in $K[X]$. Dann gäbe es ein Element $\frac{a(t)}{b(t)}$ aus $k_{0}(t)$ derart, dass die Kongruenz

$$
\left(\frac{a(t)}{b(t)}\right)^{p^{\nu}} \equiv t \quad \bmod \mathfrak{p} \quad(n \geqq \nu \geqq 1)
$$

gilt, weil $\bar{t} \overline{+} \rho_{n}=\bar{t}$ ist. Dies ist aber ein Widerspruch. Hieraus folgt sofort, dass das Polynom $X^{p^{n}}-\left(t+\rho_{n}\right)$ auch in $K[X]$ irreduzibel ist. Adjungiert man jetzt die Wurzel $\theta_{n}$ von $X^{p^{n}}-\left(t+\rho_{n}\right)=0 \mathrm{zu} K$, so ist $K_{n}=K\left(\theta_{n}\right)$ vom Grade $p^{n}$ über $K . \mathrm{Da}$

$$
\theta_{n}^{p}=\left(t+\rho_{n-1}+x^{p^{n-1} q^{-n}}\right)^{p^{-(n-1)}}=\theta_{n-1}+x^{q^{-n}} \in K_{n}
$$

ist, so ist $K_{n-1}$ ein Teilkörper von $K_{n}$. Bezeichnet man nun mit $\Re_{n}$ den Restklassenkörper von $K_{n}$, so ist die $\theta_{n}$ enthaltende Restklasse offenbar eine Nullstelle des Polynomes $X^{p^{n}}-\overline{t+\rho_{n}}$; d.h. $\Re_{n}$ ist vom Grade $p^{n}$ über $\Re$. Also ist $K_{n}$ über $K$ unverzuveigt. Bildet man nun den Vereinigungskörper $L$ von den $K_{n}(n=1$, $2, \ldots$ ), so ist $L$ über $K$ unverzweigt, also besitzt die perfekte Hülle $L$ von $L$ die Wertgruppe $W$.

Es sei nun $\mathfrak{Q}$ der Restklassenkörper von $\bar{L}$. Dann besitzt jede Restklasse $c$ aus $\because$ ein Element $c$ aus $L$ als ihren Vertreter. Da $c$ bereits zu einem passend gewählten Körper $K_{n}$ gehört, so kann man ohne Einschränkung der Allgemeinheit annehmen, dass $c$ von der Form

$$
\sum_{i=0}^{n} \frac{a_{i}(t)}{b_{i}(t)}\left(t+\rho_{n}\right)^{i p^{-n}}
$$

ist. Dabei sind die $a_{i}(t), b_{i}(t)$ Polynome von $t$ mit Koeffizienten aus $k_{0}$. Da offenbar

$$
w\left(a_{i}(t)-a_{i}\left(t+\rho_{1}\right)\right)>0, \quad w\left(b_{i}(t)-b_{i}\left(t+\rho_{1}\right)\right)>0
$$

und

3) Für $y=0$ setzen wir in üblicher Weise $w(0)=\infty$. 


$$
w\left(\left(t+\rho_{n}\right)^{p^{-n}}-\left(t+\rho_{n+1}\right)^{p^{-n}}\right)>0
$$

sind, so gehört das Element

$$
c^{\prime}=\sum_{i=0}^{n} \frac{a_{i}\left(t+\rho_{1}\right)}{b_{i}\left(t+\rho_{1}\right)}\left(t+\rho_{n+1}\right)^{i p^{-n}}=\sum_{i=0}^{m} \frac{a_{i}\left(\theta_{1}^{p}\right)}{b_{i}\left(\theta_{1}^{p}\right)} \theta_{n+1}^{i p}
$$

auch zu c. Da $k_{0}=k_{0}^{p}$ ist, so gibt es die Polynome $a_{i}^{\prime}\left(\theta_{1}\right)$ und $b_{i}^{\prime}\left(\theta_{1}\right)$ aus $K_{1}$ derart, dass

$$
a_{i}^{\prime}\left(\theta_{1}\right)^{p}=a_{i}\left(\theta_{1}^{p}\right) \quad \text { und } \quad b_{i}^{\prime}\left(\theta_{1}\right)^{p}=b_{i}\left(\theta_{1}^{p}\right) \quad(i=0,1, \ldots)
$$

gelten. Daher ist das Element $\sum_{i=0}^{m} \frac{a_{i}^{\prime}\left(\theta_{1}\right)}{b_{i}^{\prime}\left(\theta_{1}\right)} \theta_{n+1}^{i}$ die $p$-te Wurzel von $c^{\prime}$ aus $L$; d.h. der Restklassenkörper $\mathfrak{L}$ ist vollkommen.

Nun unterscheiden wir zwei Unterfälle :

$$
\text { i) } \boldsymbol{p}<\boldsymbol{q} \text { und ii) } \boldsymbol{p}>\boldsymbol{q} \text {. }
$$

Fall i). Bezeichnet man mit $t$ die $t$ enthaltende Restklasse aus $\mathfrak{L}$, so ist die $\theta_{n}$ enthaltende Restklasse aus $\mathfrak{L}$ gleich $\mathfrak{t}^{p^{-n}}$. Wir nehmen an,-dass der Durchschnitt $\bigcap_{i=0}^{\infty} \mathfrak{M}_{i}(\mathrm{t})$ nicht leer ist, und bezeichnen mit $z$ ein Element aus diesem Durchschnitt. Weil die Restklasse $t^{p^{-n}}$ aus $\mathfrak{L}$ die $p^{-n}$-te Wurzel $z^{p^{-n}}$ von $z$ enthält, so kann man

$$
z^{p^{-n}}=\theta_{n}+\sigma_{n} \quad \text { mit } w\left(\sigma_{n}\right)>0 \quad(n=0,1, \ldots)
$$

setzen $;^{4)}$ hieraus folgt ohne weiteres :

$$
z^{p^{-(n-1)}}=\theta_{n}^{p}+\sigma_{n}^{p}=\theta_{n-1}+x^{q^{-n}}+\sigma_{n}^{p}=\theta_{n-1}+\sigma_{n-1} \quad(n \geqslant 1) .
$$

Da $w\left(x^{q^{-n}}\right)=q^{-n}$ und $w\left(\sigma_{n}^{p}\right)=p w\left(\sigma_{n}\right)$ sind, so folgt aus der Struktur der Wertgruppe $W$ :

$$
w\left(x^{q^{-n}}\right) \neq w\left(\sigma_{n}^{p}\right) .
$$

Es muss also $w\left(\sigma_{n-1}\right)=\operatorname{Min}\left(q^{-n}, p w\left(\sigma_{n}\right)\right)$ sein; d.h. $w\left(\sigma_{n-1}\right) \leqq q^{-n}$. Anderseits gilt aber:

$$
w\left(\rho_{n-1}\right)=\frac{p^{n-2}}{q^{n-1}}=\left(\frac{p}{q}\right)^{n-2} \frac{1}{q}>\left(\frac{p}{q}\right)^{n-1} \frac{1}{q} \geqq w\left(\sigma_{n-1}^{p^{n-1}}\right) .
$$

Weil $\sigma_{0}=x^{q^{-1}}+\sigma_{1}^{p}, \sigma_{1}^{p}=x^{p q^{-2}}+\sigma_{2}^{p^{2}}, \ldots$, und $\sigma_{n-2}^{p^{n-2}}=x^{p^{n-2} q^{n-1}}+\sigma_{n-1}^{p^{n-1}}$ sind, so ist $\sigma_{0}=\rho_{n-1}+\sigma_{n-1}^{p_{n-1}}$, woraus

$$
w\left(\sigma_{0}\right)=w\left(\sigma_{n-1}^{p^{n-1}}\right) \leqq\left(\begin{array}{c}
p \\
q
\end{array}\right)^{n-1} \frac{1}{q}
$$

folgt. Da $\lim _{n \rightarrow \infty}\left(\frac{p}{q}\right)^{n-1} \rightarrow 0$ ist, so muss $w\left(\sigma_{0}\right)=0$ sein, was aber ein Widerspruch

4) Dabei ist $\theta_{0}=t$ gesetzt. 
ist. Also ist $\bigcap_{=0}^{\infty} \mathfrak{M}_{i}(\mathrm{t})$ leer. Nach Satz 5 besitzt $T$ kein körperisomorphes Repräsentantensystem von 2 .

Fall ii). Wegen $p>q$ konvergiert die unendliche Reihe $\sum_{i=0}^{\infty} x^{p^{i} q^{-i-1}} z u$ einem Element $y$ aus $K$. Das Element $t+y$ ist offenbar über $k_{0}$ transzendent, und $k_{0}(t+y)$ ist auch ein körperisomorphes Repräsentantensystem von $\Re$. Da

$$
t+y=t+\rho_{n}+\sum_{i=n+1}^{\infty} x^{p^{i} q^{-i-1}}
$$

ist, so ist $(t+y)^{p^{-n}}=\theta_{n}+\sum_{i=n+1}^{\infty} x^{p^{i-n} q^{-i-1}}$, wo $\sum_{i=n+1}^{\infty} x^{p^{i-n} q^{-i-1}}$ als eine konvergente Reihe aus $K$ Element aus $K$ ist. Daher ist

$$
K\left((t+y)^{p^{-n}}\right)=K\left(\theta_{n}\right)
$$

und infolgedessen ist

$$
L=\bigcup_{i=1}^{\infty} K\left((t+y)^{p^{-n}}\right) .
$$

Weil jede Restklasse aus dem Restklassenkörper $\Omega_{n}$ von $K_{n}=K\left(\theta_{n}\right)$ ein Polynom von $(t+y)^{p^{-n}}$ mit Koeffizienten aus $k_{0}(t+y)$ als einen Vertreter besitzt, so ist $k_{0}\left((t+y)^{p^{-n}}\right)$ ein körperisomorphes Repräsentantensystem von $\Re_{n}$ aus $K_{n}$. Hieraus folgt sofort, dass

$$
L_{0}=\bigcup_{i=0}^{\infty} k_{0}\left((t+y)^{p^{-n}}\right)
$$

ein körperisomorphes Repräsentantensystem des Restklassenkörpers von $L$ ist. Bekanntlich ist dabei $L_{0}$ auch ein körperisomorphes Repräsentantensystem von \&. Daher besitzt $\bar{L}$ ein körperisomorphes Repräsentantensystem des Restklassenkörpers $\mathfrak{Q}$.

Bemerkung. Der Körper $K$ enthält verschiedene körperisomorphe Repräsentantensysteme von $\Omega$, etwa $k_{0}(t)$ und $k_{0}(t+y)$.

Nach der Struktur der Wertgruppe $W$ besitzt $L$ genau ein körperisomorphes Repräsentantensystem von $\mathfrak{R}$.

\section{Mathematisches Institut,}

Universität zu Okayama 